\title{
Phase Transitions of the BTZ Black Hole in New Massive Gravity
}

\author{
Yun Soo Myung \\ Institute of Basic Science and Department of Computer Simulation, Inje University, Gimhae 621-749, Republic of Korea \\ Correspondence should be addressed to Yun Soo Myung; ysmyung@inje.ac.kr
}

Received 29 July 2015; Accepted 8 October 2015

Academic Editor: Thomas Rössler

Copyright (C) 2015 Yun Soo Myung. This is an open access article distributed under the Creative Commons Attribution License, which permits unrestricted use, distribution, and reproduction in any medium, provided the original work is properly cited. The publication of this article was funded by SCOAP ${ }^{3}$.

\begin{abstract}
We investigate thermodynamics of the BTZ black hole in new massive gravity explicitly. For $m^{2} \ell^{2}>1 / 2$ with $m^{2}$ being the mass parameter of fourth-order terms and $\ell^{2} \mathrm{AdS}_{3}$ curvature radius, the Hawking-Page phase transition occurs between the BTZ black hole and AdS (thermal) soliton. For $m^{2} \ell^{2}<1 / 2$, however, this transition unlikely occurs but a phase transition between the BTZ black hole and the massless BTZ black hole is possible to occur. We may call the latter the inverse Hawking-Page phase transition and this transition is favored in the new massive gravity.
\end{abstract}

\section{Introduction}

A black hole could be rendered thermodynamically stable by placing it in four-dimensional anti-de Sitter $\left(\mathrm{AdS}_{4}\right)$ spacetimes because $\mathrm{AdS}_{4}$ spacetimes play the role of a confining box. Then, it is a natural question to ask how a stable black hole with positive heat capacity could emerge from thermal radiation through a phase transition. This was known to be the Hawking-Page (HP) phase transition between thermal radiation (TR) and Schwarzschild-AdS black hole (SAdS) $[1,2]$. It has shown one typical example of the first-order phase transition (TAdS $\rightarrow$ small SAdS $\rightarrow$ large SAdS) in the gravitational system. In the last two decades, its higher dimensional extension and its holographic duality to confinement-deconfinement transition were the hottest issues [3].

In order to study the HP phase transition in Einstein gravity, we need to know the Arnowitt-Deser-Misner (ADM) mass [4], the Hawking temperature, and the BekensteinHawking (BH) entropy. These are combined to give the onshell free energy in canonical ensemble which determines the global thermodynamic stability. The other important quantity is the heat capacity which determines the local thermodynamic stability. Employing the Euclidean action formalism, one easily finds these quantities [5]. However, a complete computation of the thermodynamic quantities was limited in fourth-order gravity because one has encountered some difficulty computing their conserved quantities in asymptotically AdS spacetimes.

In three dimensions, either third-order gravity (topologically massive gravity [6-8]) or the fourth-order gravity (new massive gravity [9]) is essential to describe spin2 graviton because the Einstein gravity is a gauge theory without propagating degrees of freedom. Recently, there was significant progress on computation of mass and thermodynamic quantities by using the Abbott-Deser-Tekin (ADT) method [10-12]. One has to recognize that all ADT thermodynamic quantities except the Hawking temperature depend on a mass parameter $m^{2}$. Hence, for $m^{2} \ell^{2}>1 / 2$, all thermodynamic properties are dominantly determined by Einstein gravity, while, for $m^{2} \ell^{2}<1 / 2$, all thermodynamic properties are dominantly determined by purely fourth-order curvature term. More recently, it was shown that the HP phase transition (thermal soliton $\rightarrow$ BTZ black hole) occurs for $m^{2} \ell^{2}>1 / 2$ in new massive gravity by computing offshell free energies of black hole and soliton [13]. However, the role of the massless BTZ black hole was missed. The former can be completely understood if the massless BTZ black hole is introduced as a mediator. Furthermore, the present is a turnaround time to explore $m^{2} \ell^{2}<1$ case of black hole thermodynamics if one wishes to study the black hole thermodynamics by employing the massive gravity theory.

On the other hand, we would like to mention that the stability condition of the BTZ black hole in the new massive 
gravity turned out to be $m^{2} \ell^{2}>1 / 2$ regardless of the horizon size $r_{+}$, while the instability condition is given by $m^{2} \ell^{2}<1 / 2$ [14]. For $m^{2} \ell^{2}<1 / 2$, the BTZ black hole is thermodynamically unstable because of $C_{\mathrm{ADT}}<0$ and $F_{\mathrm{ADT}}^{\mathrm{on}}>0$ and it is classically unstable against the metric perturbations. The latter indicates perturbative instability of the BTZ black hole that arose from the massiveness of graviton. It implies a deep connection between thermodynamic instability and classical instability for the BTZ black hole only for the new massive gravity [15]. Also, it suggests that the phase transition for $m^{2} \ell<1 / 2$ is quite different from that of $m^{2} \ell>1 / 2$ case. Here, we wish to explore the presumed phase transition and it will be compared with the Hawking-Page phase transition for $m^{2} \ell>1 / 2$ case.

\section{Thermodynamics of the BTZ Black Hole}

We introduce the new massive gravity (NMG) composed of the Einstein-Hilbert action with a cosmological constant $\lambda$ and fourth-order curvature terms [9]:

$$
\begin{aligned}
S_{\mathrm{NMG}} & =S_{\mathrm{EH}}+S_{\mathrm{FOT}}, \\
S_{\mathrm{EH}} & =\frac{1}{16 \pi G} \int d^{3} x \sqrt{-g}(R-2 \lambda), \\
S_{\mathrm{FOT}} & =-\frac{1}{16 \pi G m^{2}} \int d^{3} x \sqrt{-g}\left(R_{\mu \nu} R^{\mu \nu}-\frac{3}{8} R^{2}\right),
\end{aligned}
$$

where $G$ is a three-dimensional Newton constant and $m^{2}$ a mass parameter with mass dimension 2. In the limit of $m^{2} \rightarrow$ $\infty, S_{\mathrm{NMG}}$ recovers the Einstein gravity, while $S_{\mathrm{NMG}}$ reduces to purely fourth-order gravity in the limit of $m^{2} \rightarrow 0$. The Einstein equation is given by

$$
R_{\mu \nu}-\frac{1}{2} g_{\mu \nu} R+\lambda g_{\mu \nu}-\frac{1}{2 m^{2}} K_{\mu \nu}=0,
$$

where

$$
\begin{aligned}
K_{\mu \nu}= & 2 \square R_{\mu \nu}-\frac{1}{2} \nabla_{\mu} \nabla_{\nu} R-\frac{\square R}{2} g_{\mu \nu}+4 R_{\mu \rho \nu \sigma} R^{\rho \sigma} \\
& -\frac{3 R}{2} R_{\mu \nu}-R_{\rho \sigma}^{2} g_{\mu \nu}+\frac{3 R^{2}}{8} g_{\mu \nu} .
\end{aligned}
$$

The BTZ black hole solution to $(4)$ is given by $[16,17]$

$$
\begin{aligned}
d s_{\mathrm{BTZ}}^{2} & =\bar{g}_{\mu \nu} d x^{\mu} d x^{\nu}=-f(r) d t^{2}+\frac{d r^{2}}{f(r)}+r^{2} d \phi^{2}, \\
f(r) & =-M+\frac{r^{2}}{\ell^{2}}
\end{aligned}
$$

when satisfying a condition of $1 / \ell^{2}+\lambda+1 /\left(4 m^{2} \ell^{4}\right)=0$ with $\ell^{2}$ being the curvature radius of $\mathrm{AdS}_{3}$ spacetimes. Here, $M$ is related to the ADM mass of black hole. The horizon radius $r_{+}$ is determined by the condition of $f\left(r_{+}\right)=0$.

On the other hand, the linearized equation to (4) upon choosing the transverse-traceless gauge of $\bar{\nabla}^{\mu} h_{\mu \nu}=0$ and $h^{\mu}{ }_{\mu}=0$ leads to the fourth-order linearized equation for the metric perturbation $h_{\mu \nu}$ :

$$
\left(\bar{\nabla}^{2}-2 \Lambda\right)\left[\bar{\nabla}^{2}-2 \Lambda-\mathscr{M}^{2}\left(m^{2}\right)\right] h_{\mu \nu}=0, \quad \Lambda=-\frac{1}{\ell^{2}},
$$

which might imply the two second-order linearized equations

$$
\begin{aligned}
\left(\bar{\nabla}^{2}-2 \Lambda\right) h_{\mu \nu} & =0, \\
{\left[\bar{\nabla}^{2}-2 \Lambda-M^{2}\left(m^{2}\right)\right] h_{\mu \nu} } & =0,
\end{aligned}
$$

where the mass squared $\mathscr{M}^{2}$ of massive spin-2 graviton is given by

$$
\mathscr{M}^{2}\left(m^{2}\right)=m^{2}-\frac{1}{2 \ell^{2}} \longrightarrow \frac{M^{2}}{m^{2}}=1-\frac{1}{2 m^{2} \ell^{2}} .
$$

Equation (9) describes massive graviton with $2(6-4=$ 2) DOF propagating around the BTZ black hole under the gauge, while (8) indicates nonpropagating spin-2 graviton in the Einstein gravity. This explains clearly why the NMG describes massive graviton with $2 \mathrm{DOF}$. The presence of $S_{\mathrm{FOT}}$ distinguishes the NMG from the Einstein gravity because it generates 2 DOF. At this stage, we briefly mention the stability of the BTZ black hole in the NMG. The stability condition of the BTZ black hole in the NMG turned out to be $m^{2} \ell^{2}>$ $1 / 2\left(\mathscr{M}^{2}>0\right)$ regardless of the horizon size $r_{+}$, while the instability condition is given by $m^{2} \ell^{2}<1 / 2\left(\mathscr{M}^{2}<0\right)$ [14]. This is valid for the NMG, not for the Einstein gravity.

Now we derive all thermodynamic quantities. The Hawking temperature is found to be

$$
T_{\mathrm{H}}=\frac{f^{\prime}\left(r_{+}\right)}{4 \pi}=\frac{r_{+}}{2 \pi \ell^{2}},
$$

which is the same for the Einstein gravity. Using the ADT method, one could derive the mass [18], heat capacity, entropy [19], and on-shell free energy:

$$
\begin{aligned}
& M_{\mathrm{ADT}}=\frac{\mathscr{M}^{2}}{m^{2}} M, \\
& C_{\mathrm{ADT}}=\frac{\mathscr{M}^{2}}{m^{2}} C, \\
& S_{\mathrm{ADT}}=\frac{\mathscr{M}^{2}}{m^{2}} S_{\mathrm{BH}}, \\
& F_{\mathrm{ADT}}^{\mathrm{on}}=\frac{\mathscr{M}^{2}}{m^{2}} F^{\mathrm{on}} .
\end{aligned}
$$

For $G=1 / 8$, thermodynamic quantities in Einstein gravity are given by [20-22]

$$
\begin{aligned}
M & =\frac{r_{+}^{2}}{\ell^{2}}, \\
C & =4 \pi r_{+}, \\
S_{\mathrm{BH}} & =4 \pi r_{+}, \\
F^{\mathrm{on}} & =M-T_{\mathrm{H}} S_{\mathrm{BH}}=-\frac{r_{+}^{2}}{\ell^{2}}=-M,
\end{aligned}
$$


which are positive regardless of the horizon size $r_{+}$except that the free energy is negative. This means that the BTZ black hole is thermodynamically stable in Einstein gravity. Here, we check that the first law of thermodynamics is satisfied as

$$
d M_{\mathrm{ADT}}=T_{\mathrm{H}} d S_{\mathrm{ADT}},
$$

as in Einstein gravity

$$
d M=T_{\mathrm{H}} d S_{\mathrm{BH}},
$$

where " $d$ " denotes the differentiation with respect to the horizon size $r_{+}$only. Importantly, we note that in the limit of $m^{2} \rightarrow \infty$ we recover thermodynamics of the BTZ black hole in Einstein gravity, while in the limit of $m^{2} \rightarrow 0$ we recover the black hole thermodynamics in purely fourthorder gravity. The latter is similar to recovering the thirdorder terms of conformal Chern-Simons gravity from the topologically massive gravity [23] and conformal gravity from the Einstein-Weyl gravity [15, 24].

It is well known that the local thermodynamic stability is determined by the positive heat capacity $\left(C_{\mathrm{ADT}}>0\right)$ and the global stability is determined by the negative free energy $\left(F_{\mathrm{ADT}}^{\mathrm{on}}<0\right)$. Therefore, we propose that the thermodynamic stability is determined by the sign of the heat capacity while the phase transition is mainly determined by the sign of the free energy.

To investigate a phase transition, we introduce the thermal soliton (TSOL) whose thermodynamic quantities are given by [13]

$$
\begin{aligned}
& M_{\mathrm{ADT}}^{\mathrm{TSOL}}\left(m^{2}\right)=\frac{\mathscr{M}^{2}}{m^{2}} M^{\mathrm{TSOL}}, \\
& F_{\mathrm{ADT}}^{\mathrm{TSOL}}\left(m^{2}\right)=\frac{\mathscr{M}^{2}}{m^{2}} F^{\mathrm{TSOL}},
\end{aligned}
$$

where

$$
\begin{aligned}
& M^{\mathrm{TSOL}}=1, \\
& F^{\mathrm{TSOL}}=-1,
\end{aligned}
$$

with $G=1 / 8$. We note that a factor of $\mathscr{M}^{2} / \mathrm{m}^{2}$ was missed in [15]. The TSOL corresponds to the spacetime picture of the NS-NS vacuum state [25].

Furthermore, we need the massless BTZ black hole (MBTZ) whose thermodynamic quantities all are zero as $[21,26]$

$$
\begin{gathered}
M_{\mathrm{ADT}}^{\mathrm{MBTZ}}=0, \\
F_{\mathrm{ADT}}^{\mathrm{MBTZ}}=0 .
\end{gathered}
$$

The MBTZ is called the spacetime picture of the $R-R$ vacuum state.

In addition to a global mass parameter $\mathrm{m}^{2}$, we introduce five parameters to describe the phase transition in NMG. These are included as

(i) $M$ : order parameter, (ii) $T_{\mathrm{H}}(M)$ : order parameter (on-shell temperature),

(iii) $T$ : control parameter (off-shell temperature),

(iv) $F_{\mathrm{ADT}}^{\mathrm{on}}\left(m^{2}, M\right)$ : increasing (decreasing) black hole via equilibrium process,

(v) $F_{\mathrm{ADT}}^{\mathrm{off}}\left(m^{2}, M, T\right)$ : increasing (decreasing) black hole via nonequilibrium process,

where off-shell (on-shell) means equilibrium (nonequilibrium) configurations. In general, the equilibrium process implies a reversible process, while the nonequilibrium process implies an irreversible process. The off-shell free energy corresponds to a generalized free energy which is similar to a temperature-dependent scalar potential $V(\varphi, T)$ for a simple model of thermal phase transition where $\varphi$ is the order parameter and $T$ is a control parameter.

\section{Phase Transitions}

3.1. HP Phase Transition. Before we proceed, we understand intuitively how the original HP transition occurs between Schwarzschild-AdS $\mathrm{A}_{4}$ black hole (SAdS) and thermal radiation (TR). For $G_{4}=1$, the ADM mass, Hawking temperature, and the Bekenstein-Hawking entropy are given by

$$
\begin{aligned}
M_{\text {SAdS }}\left(r_{+}\right) & =\frac{1}{2}\left(r_{+}+\frac{r_{+}^{3}}{l^{2}}\right), \\
T_{\mathrm{H}}\left(r_{+}\right) & =\frac{1}{4 \pi}\left(\frac{1}{r_{+}}+\frac{3 r_{+}}{l^{2}}\right), \\
S_{\mathrm{BH}} & =\pi r_{+}^{2} .
\end{aligned}
$$

In addition, the heat capacity and on-shell free energy are given by

$$
\begin{aligned}
& C_{\text {SAdS }}\left(r_{+}\right)=2 \pi r_{+}^{2}\left(\frac{3 r_{+}^{2}+l^{2}}{3 r_{+}^{2}-l^{2}}\right), \\
& F_{\text {SAdS }}^{\text {on }}\left(r_{+}\right)=\frac{r_{+}}{4}\left(1-\frac{r_{+}^{2}}{l^{2}}\right),
\end{aligned}
$$

where $C_{\text {SAdS }}$ blows up at $r_{+}=r_{*}=l / \sqrt{3}$ (heat capacity is changed from $-\infty$ to $\infty$ at $r_{+}=r_{*}$ ). The critical temperature $T_{c}=\left.T_{H}\left(r_{+}\right)\right|_{r_{+}=r_{c}}=1 / \pi l$ is determined from the condition of $F_{\text {SAdS }}^{\text {on }}\left(r_{+}\right)=0$ for $r_{+}=r_{c}=l$. The TR is located at $r_{+}=0$ in this picture.

In studying thermodynamic stability, two relevant quantities are the heat capacity $C_{\text {SAdS }}$ which determines thermally local stability (instability) for $C_{\text {SAdS }}>0\left(C_{\text {SAdS }}<0\right)$ and on-shell free energy $F_{\text {SAdS }}^{\text {on }}$ which determines the thermally global stability (instability) for $F_{\text {SAdS }}^{\text {on }}<0$ ( $\left.F_{\text {SAdS }}^{\text {on }}>0\right)$. SAdS is thermodynamically stable only if $C_{\text {SAdS }}>0$ and $F_{\text {SAdS }}^{\text {on }}<0$. For simplicity, we choose $l=1$. We observe that the onshell free energy (thick curve in Figure 1) is maximum at $r_{+}=r_{*}=0.57$ and zero at $r_{+}=r_{c}=1$ which determines the critical temperature. For $r_{+}>r_{c}$, one finds negative free energy. 


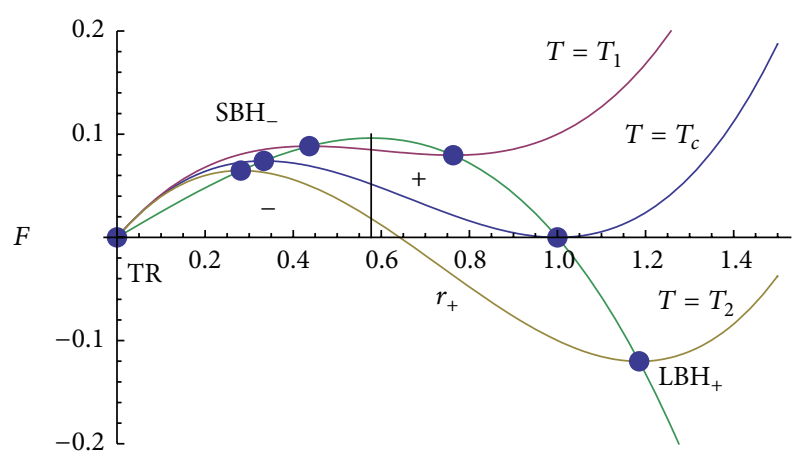

FIGURE 1: Hawking-Page phase transition for the SAdS with $l=1$ : the thick curve represents the on-shell free energy $F_{\mathrm{SAdS}}^{\text {on }}\left(r_{+}\right)$, while three thin curves denote the off-shell free energy $F_{\text {SAdS }}^{\text {off }}\left(r_{+}, T\right)$ with three temperatures $T=T_{1}=0.9 \pi^{-1}\left(<T_{c}\right), T_{c}=\pi^{-1}$, and $T_{2}=$ $1.1 \pi^{-1}\left(>T_{c}\right) .-(+)$ denotes negative (positive) heat capacity. $\mathrm{SBH}_{-}$ is bounded in the right by the line at $r_{+}=r_{*}=0.57$.

In order to investigate the HP phase transition, one has to introduce the off-shell free energy as a function of $r_{+}$and $T$ :

$$
F_{\text {SAdS }}^{\text {off }}\left(r_{+}, T\right)=M_{\text {SAdS }}\left(r_{+}\right)-T S_{\mathrm{BH}}\left(r_{+}\right) \text {, }
$$

where $T$ plays a role of control parameter for taking a phase transition.

For $T=T_{2}>T_{c}$, the process of phase transition is shown in Figure 1 explicitly. In this case, one starts with TR $(\bullet)$ at $r_{+}=0$ in AdS space and a small black hole $\left(\bullet: \mathrm{SBH}_{-}\right)$appears at $r_{+}=0.28$. Here, $\mathrm{SBH}_{-}$denotes unstable small black hole with $C_{\text {SAdS }}<0$ and $F_{\text {SAdS }}^{\text {on }}>0$. This plays a role of the mediator. Then, since the heat capacity changes from $-\infty$ to $\infty$ at $r_{+}=r_{*}$, the large black hole $\left(\bullet: \mathrm{LBH}_{+}\right)$finally comes out as a stable object at $r_{+}=1.19$. Here, $\mathrm{LBH}_{+}$represents a globally stable black hole because of $C_{\text {SAdS }}>0$ and $F_{\text {SAdS }}^{\text {on }}<0$. Actually, there is a change of the dominance at the critical temperature $T=T_{c}$ : from TR to SAdS [1]. This is called the HP phase transition as a typical example of the first-order transition in the gravitational system: $\mathrm{TR} \rightarrow \mathrm{SBH}_{-} \rightarrow \mathrm{LBH}_{+}$.

For $T=T_{1}\left(<T_{c}\right)$, the free energy $F_{\text {SAdS }}^{\text {on }}(0)=0$ of TR is the lowest state, while, for $T=T_{2}\left(>T_{c}\right)$ case, the lowest state is the free energy $F_{\text {SAdS }}^{\text {on }}(1.19)<0$ for the large black hole. Hence, for $T_{1}<T_{c}$, the ground state is TR, whereas, for $T_{2}>T_{c}$, the ground state is $\mathrm{LBH}_{-}$. There is no phase transition for $T=T_{1}$.

3.2. HP Transition in NMG. The off-shell free energy for the $\mathrm{BTZ}$ black hole is given by

$$
F_{\text {ADT }}^{\mathrm{off}}\left(m^{2}, M, T\right)=\frac{\mathscr{M}^{2}}{m^{2}}(M-4 \pi \ell T \sqrt{M}) .
$$

On the other hand, the off-shell free energy for TSOL takes the form $[13,22]$

$$
F_{\mathrm{TSOL}}^{\mathrm{off}}\left(m^{2}, M\right)=\frac{\mathscr{M}^{2}}{m^{2}}(-M-2 \sqrt{-M}) .
$$

It is worth noting that the author has claimed that the phase transition from TSOL to BTZ is possible to occur without

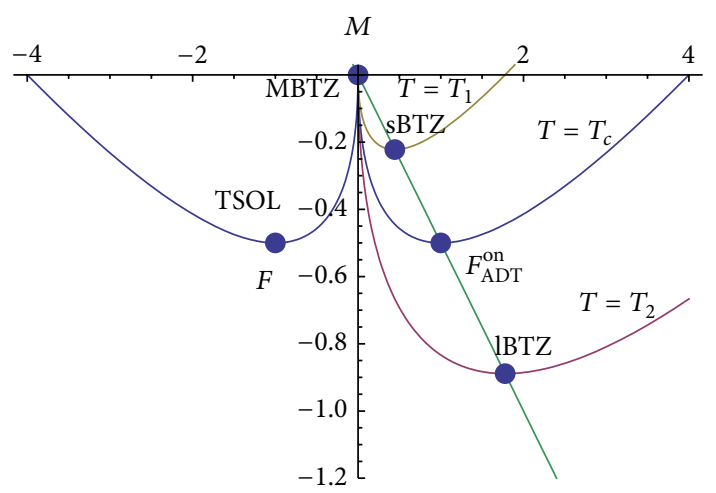

FIGURE 2: On-shell free energy $F_{\mathrm{ADT}}^{\mathrm{on}}\left(m^{2}=1, M\right)=-M / 2$ for the $\mathrm{BTZ}$ and $F_{\mathrm{ADT}}^{\mathrm{TSOL}}\left(m^{2}=1\right)=-0.5$ for TSOL with $G_{3}=1 / 8$ and $\ell=1$. Three off-shell free energies for the BTZ are given by $F_{\mathrm{ADT}}^{\text {off }}\left(m^{2}=\right.$ $1, M, T)$ for $T=T_{1}=1 / 3 \pi, T_{c}=1 / 2 \pi$, and $T_{2}=2 / 3 \pi$. On the other hand, the off-shell free energy for TSOL is $F_{\text {TSOL }}^{\text {off }}$. They are connected at the point of $M=0$. Here, the MBTZ located at $M=0$ plays a role of the mediator like $\mathrm{SBH}_{-}$.

introducing (23) [21]. However, the construction of (23) is necessary to show how the transition from TSOL to BTZ occurs nicely [22].

Here, we would like to mention that, for $\mathscr{M}^{2}>0$, the BTZ under consideration are thermally stable because the heat capacity is always positive. Hence, the free energy plays a key role in studying a phase transition between two gravitational configurations. We emphasize that two on-shell free energies $F_{\mathrm{ADT}}^{\mathrm{on}}$ and $F^{\mathrm{TSOL}}=-1 / 2$ are disconnected to each other. To discuss the phase transition with $\mathscr{M}^{2}>$ $0\left(m^{2}=1>1 / 2\right)$, it would be better to examine two offshell free energies (22) and (23). One finds from Figure 2 that, for $T=T_{1}$, the TSOL $(\bullet)$ is more favorable than the small BTZ $(\bullet)$, while, for $T=T_{2}$, the large BTZ $(\bullet)$ is more favorable than TSOL $(\bullet)$. This observation suggests a phase transition (TSOL $\rightarrow \mathrm{MBTZ} \rightarrow \mathrm{BTZ}$ ) for $T>T_{c}$. Two off-shell free energies are connected at the point $M=0$. At $T=T_{c}$, the transition from TSOL to BTZ black hole through MBTZ is possible to occur. For $T=T_{1}<T_{c}$, the TSOL dominates because of $F^{\mathrm{TSOL}}<F_{\mathrm{ADT}}^{\mathrm{on}}$, while, for $T=T_{2}>T_{c}$, the BTZ dominates because of $F^{\mathrm{TSOL}}>F_{\mathrm{ADT}}^{\mathrm{on}}$. This indicates that a change of dominance occurs at the critical temperature $T=T_{c}$ [21]. Importantly, this transition could be regarded really as a HP transition because the MBTZ plays a role of the mediator like $\mathrm{SBH}_{-}$in the previous $\mathrm{HP}$ phase transition [15]. A difference is that the MBTZ is a single extremal state (see Figure 2), whereas $\mathrm{SBH}_{-}$has three states depending on $T$ (see Figure 1). A similarity is that they all are the highest states.

3.3. Inverse HP Transition in NMG. Generally, increasing black hole $(\searrow)$ is induced by absorbing radiation in the heat reservoir, while decreasing black hole $(\swarrow)$ is done by Hawking radiation as evaporation process. The former describes the HP phase transition, whereas the latter denotes the inverse Hawking-Page phase transition (IHP). 


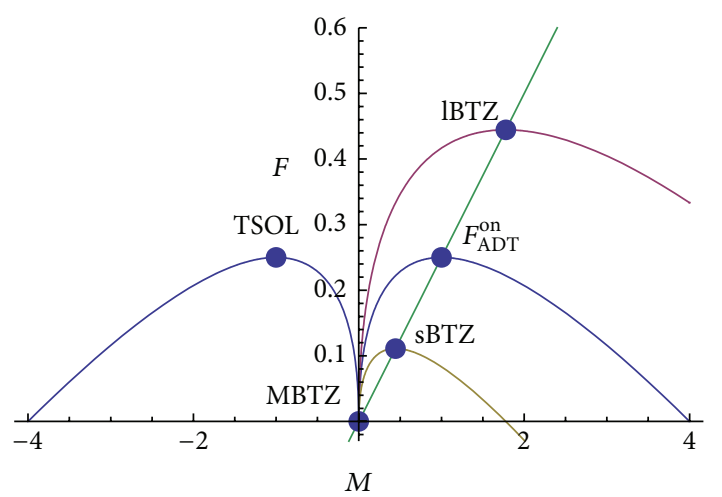

FIGURE 3: On-shell free energy $F_{\mathrm{ADT}}^{\mathrm{on}}\left(m^{2}=0.4, M\right)=0.25 \mathrm{M}$ for the BTZ and $F_{\mathrm{ADT}}^{\mathrm{TSOL}}\left(m^{2}=0.4\right)=0.25$ for TSOL. Three off-shell free energies for the BTZ are given by $F_{\mathrm{ADT}}^{\mathrm{off}}\left(m^{2}=0.4, M, T\right)$ for $T=T_{1}=$ $1 / 3 \pi, T_{c}=1 / 2 \pi$, and $T_{2}=2 / 3 \pi$. On the other hand, the off-shell free energy for TSOL is $F_{\mathrm{TSOL}}^{\text {off }}\left(m^{2}=0.4, M\right)$. Here, we observe that the MBTZ located at $M=0$ is the ground state.

For $\mathscr{M}^{2}<0$ case, fourth-order curvature terms (3) contribute dominantly to black hole thermodynamics. In this case, the heat capacity of the BTZ is negative and the on-shell free energy is positive, which means that the BTZ is thermodynamically unstable. This is consistent with the classical instability of the BTZ for $m^{2}<1 / 2$ [14]. The other quantities of ADT mass and entropy are negative, which may raise a problem in obtaining consistent black hole thermodynamics. To avoid this problem, the author in [13] has required $\mathscr{M}^{2}>0$. However, at this moment, we could not understand why this problem arises in the ADT approach to the black hole thermodynamics in the NMG. Instead, it would be better to make progress on the phase transition because this is a main feature of the BTZ in the NMG. Here, we wish to know whether a phase transition from the BTZ to TSOL is possible to occur in NMG for $\mathscr{M}^{2}<0\left(\mathrm{~m}^{2}=\right.$ $0.4<1 / 2$ ). We find from Figure 3 that the MBTZ located at $M=0$ is always more favorable than BTZ and TSOL because of $F_{\mathrm{MBTZ}}<F_{\mathrm{ADT}}^{\mathrm{on}}\left(m^{2}=0.4, M\right), F^{\mathrm{TSOL}}\left(m^{2}=0.4\right)$. In other words, it corresponds to the ground state. In this case, we might not define a possible phase transition between TSOL and BTZ because the ground state is given by the MBTZ. A possible transition is the IHP (BTZ $\rightarrow$ MBTZ) [21], whereas the TSOL is located at the left branch. This may be possible because the MBTZ is considered as an extremal black hole without size.

\section{Discussions}

We have investigated thermodynamics of the BTZ black hole in new massive gravity completely. We have confirmed that, for $m^{2} \ell^{2}>1 / 2$, the Hawking-Page phase transition occurs between the BTZ black hole and thermal soliton by introducing the massless BTZ black hole. Here, the massless BTZ black hole plays a role of the mediator like $\mathrm{SBH}_{-}$in the original Hawking-Page phase transition and it is the highest state.
On the other hand, for $m^{2} \ell^{2}<1 / 2$, this transition unlikely occurs but a phase transition between the BTZ black hole and the massless BTZ black hole is possible to occur. In this case, the massless BTZ black hole is the ground state. We call the latter the inverse Hawking-Page phase transition and this transition is favored in the new massive gravity. This completes phase transitions of the BTZ black hole in the new massive gravity.

\section{Conflict of Interests}

The author declares that there is no conflict of interests regarding the publication of this paper.

\section{Acknowledgment}

This work was supported by the 2015 Inje University research grant.

\section{References}

[1] S. W. Hawking and D. N. Page, "Thermodynamics of black holes in anti-de Sitter space," Communications in Mathematical Physics, vol. 87, no. 4, pp. 577-588, 1983.

[2] J. D. Brown, J. Creighton, and R. B. Mann, "Temperature, energy, and heat capacity of asymptotically anti-de Sitter black holes," Physical Review D, vol. 50, no. 10, pp. 6394-6403, 1994.

[3] E. Witten, "Anti-de Sitter space, thermal phase transition, and confinement in gauge theories," Advances in Theoretical and Mathematical Physics, vol. 2, pp. 505-532, 1998.

[4] R. Arnowitt, S. Deser, and C. W. Misner, "Republication of: the dynamics of general relativity," General Relativity and Gravitation, vol. 40, no. 9, pp. 1997-2027, 2008.

[5] C. V. Johnson, D-Branes, Cambridge University Press, Cambridge, UK, 2003.

[6] S. Deser, R. Jackiw, and S. Templeton, "Topologically massive Gauge theories," Annals of Physics, vol. 140, no. 2, pp. 372-411, 1982.

[7] S. Deser, R. Jackiw, and S. Templeton, "Erratum," Annals of Physics, vol. 185, no. 2, p. 406, 1988.

[8] S. Deser, R. Jackiw, and S. Templeton, “Topologically massive Gauge theories," Annals of Physics, vol. 281, no. 1-2, pp. 409-449, 2000.

[9] E. A. Bergshoeff, O. Hohm, and P. K. Townsend, "Massive gravity in three dimensions," Physical Review Letters, vol. 102, no. 20, Article ID 201301, 2009.

[10] L. F. Abbott and S. Deser, "Stability of gravity with a cosmological constant," Nuclear Physics B, vol. 195, no. 1, pp. 76-96, 1982.

[11] S. Deser and B. Tekin, "Energy in generic higher curvature gravity theories," Physical Review D, vol. 67, no. 8, Article ID 084009, 2003.

[12] W. Kim, S. Kulkarni, and S.-H. Yi, "Quasi-local conserved charges in covariant theory of gravity," Physical Review Letters, vol. 111, Article ID 081101, 2013.

[13] S.-J. Zhang, "Hawking-page phase transition in new massive gravity," Physics Letters B, vol. 747, pp. 158-163, 2015.

[14] T. Moon and Y. S. Myung, "Gregory-Laflamme instability of the BTZ black hole in new massive gravity," Phys. Rev. D, vol. 88, no. 12, Article ID 124014, 2013. 
[15] Y. S. Myung and T. Moon, "Thermodynamic and classical instability of AdS black holes in fourth-order gravity," Journal of High Energy Physics, vol. 2014, no. 4, article 58, 2014.

[16] M. Bañados, C. Teitelboim, and J. Zanelli, "Black hole in threedimensional spacetime," Physical Review Letters, vol. 69, no. 13, pp. 1849-1851, 1992.

[17] M. Bañados, M. Henneaux, C. Teitelboim, and J. Zanelli, "Geometry of the $2+1$ black hole," Physical Review D, vol. 48, no. 4, pp. 1506-1525, 1993.

[18] G. Clement, "Warped $\mathrm{AdS}_{3}$ black holes in new massive gravity," Classical and Quantum Gravity, vol. 26, no. 10, Article ID 105015, 2009.

[19] W. Kim, S. Kulkarni, and S.-H. Yi, "Conserved quantities and Virasoro algebra in new massive gravity," Journal of High Energy Physics, vol. 2013, no. 5, article 041, 2013.

[20] Y. S. Myung, "No Hawking-Page phase transition in three dimensions," Physics Letters B, vol. 624, no. 3-4, pp. 297-303, 2005.

[21] Y. S. Myung, "Phase transition between the BTZ black hole and AdS space," Physics Letters B, vol. 638, no. 5-6, pp. 515-518, 2006.

[22] M. Eune, W. Kim, and S.-H. Yi, "Hawking-Page phase transition in BTZ black hole revisited," Journal of High Energy Physics, vol. 2013, no. 3, article 020, 2013.

[23] A. Bagchi, S. Detournay, D. Grumiller, and J. Simon, "Cosmic evolution from phase transition of 3-dimensional flat space," Physical Review Letters, vol. 111, no. 18, Article ID 181301, 5 pages, 2013.

[24] H. Lü, Y. Pang, C. N. Pope, and J. F. Vázquez-Poritz, "AdS and Lifshitz black holes in conformal and Einstein-Weyl gravities," Physical Review D, vol. 86, no. 4, Article ID 044011, 2012.

[25] J. M. Maldacena and A. Strominger, "AdS 3 black holes and a stringy exclusion principle," Journal of High Energy Physics, vol. 1998, no. 12, article 005, 1998.

[26] X. P. Rao, B. Wang, and G. H. Yang, "Quasinormal modes and phase transition of black holes," Physics Letters B, vol. 649, no. 5-6, pp. 472-477, 2007. 

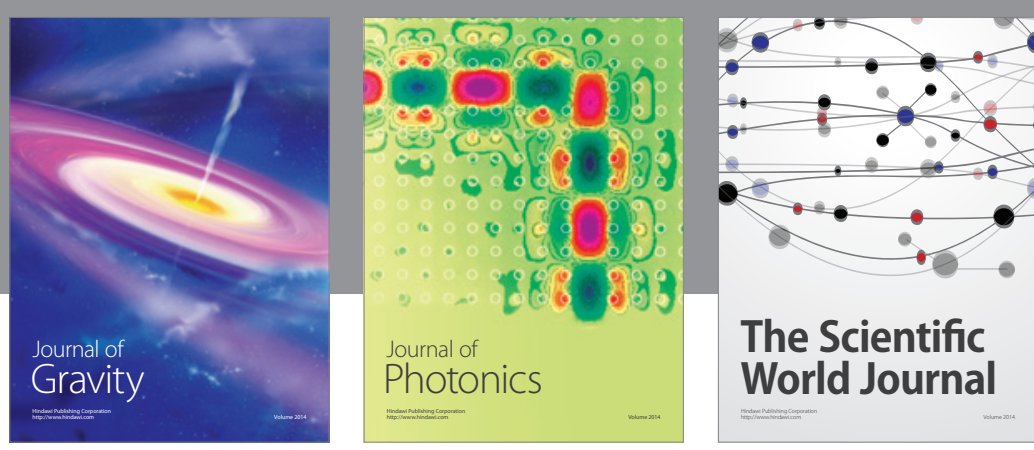

The Scientific World Journal
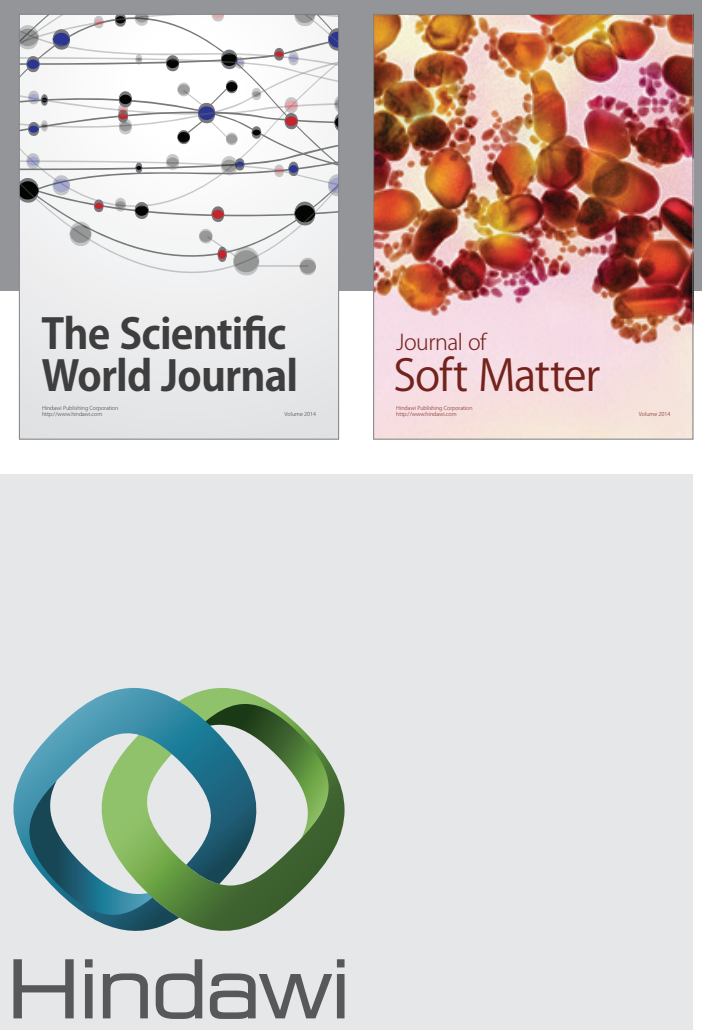

Submit your manuscripts at

http://www.hindawi.com

nternational Journal of

Statistical Mechanics
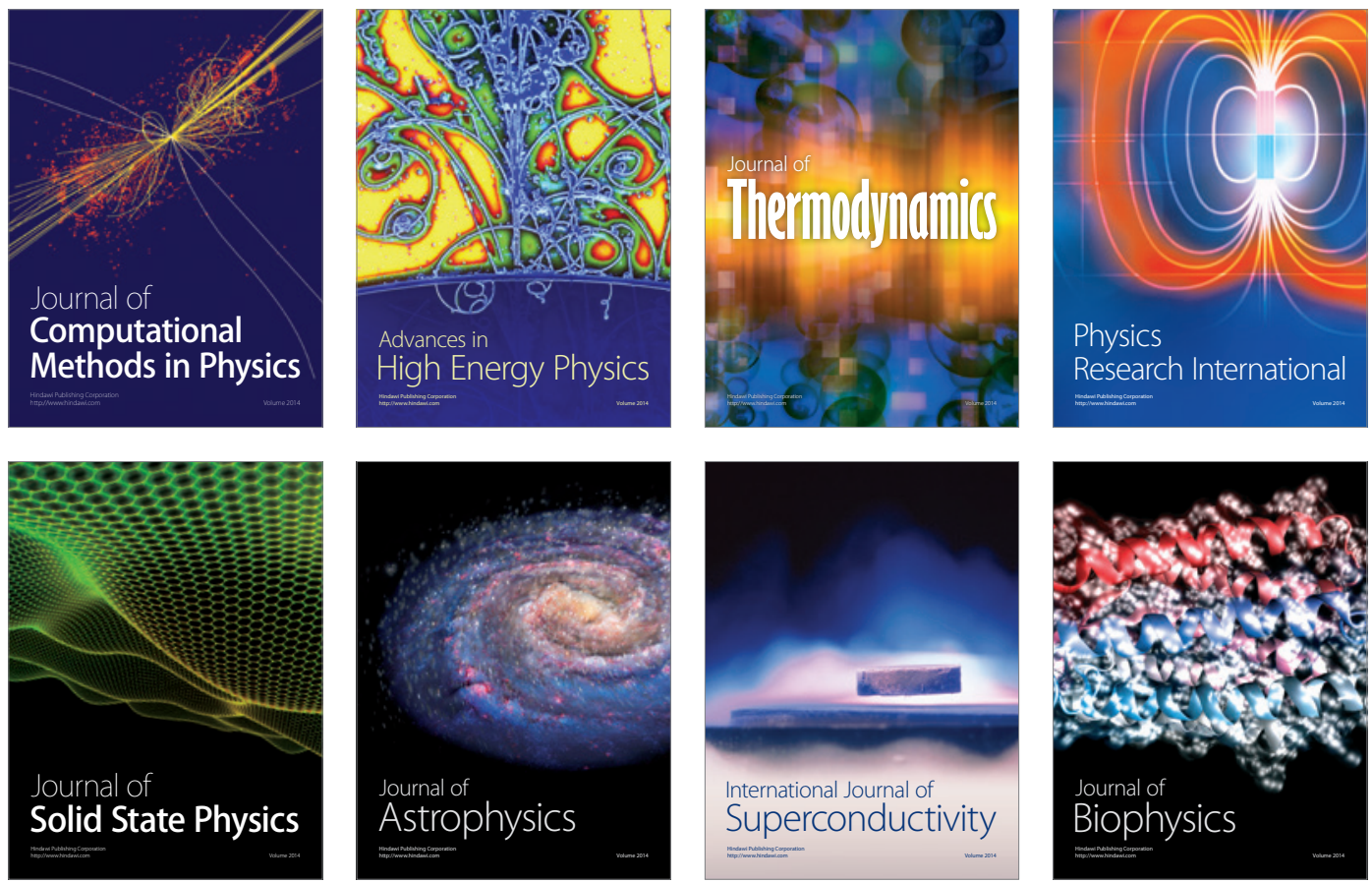
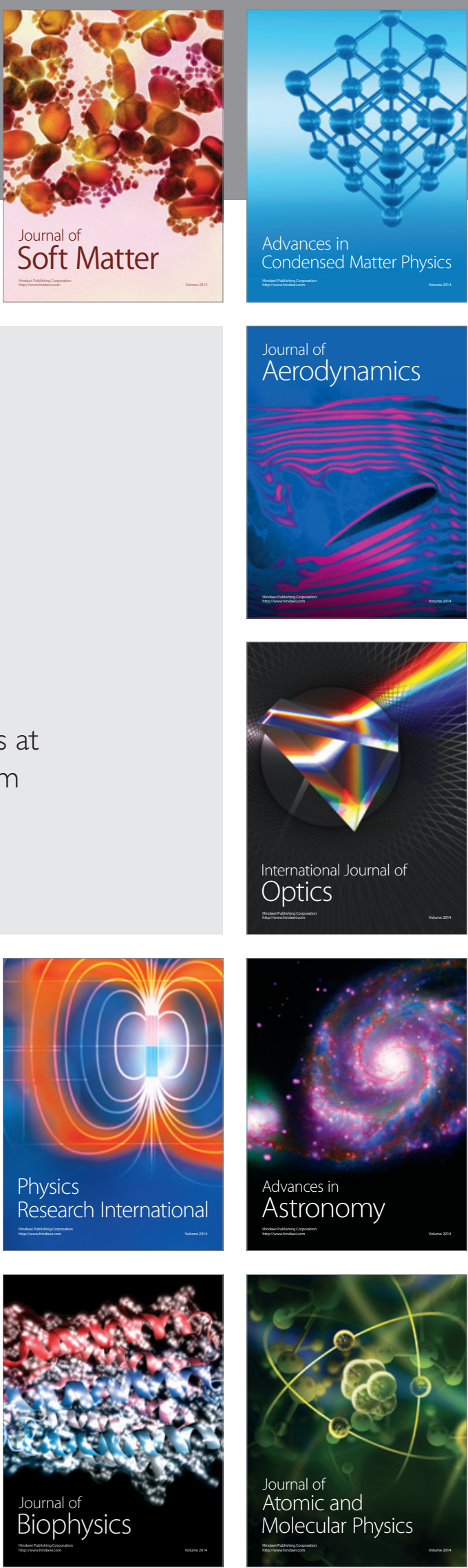